\title{
The role of tolvaptan in managing hyponatremia in small cell lung cancer patients with SIADH: a retrospective study of 23 cases
}

\author{
Peng Ren, Qiuan Yang^ \\ Department of Radiation Oncology, Qilu Hospital, Cheeloo College of Medicine, Shandong University, Jinan, China \\ Contributions: (I) Conception and design: All authors; (II) Administrative support: Q Yang; (III) Provision of study materials or patients: All authors; \\ (IV) Collection and assembly of data: P Ren; (V) Data analysis and interpretation: P Ren; (VI) Manuscript writing: All authors; (VII) Final approval \\ of manuscript: All authors. \\ Correspondence to: Qiuan Yang. Department of Radiation Oncology, Qilu Hospital, Cheeloo College of Medicine, Shandong University, Jinan 250012, \\ China. Email: yangqiuan9966@126.com.
}

Background: Existing studies have shed light on the treatment of small cell lung cancer (SCLC), but data on tolvaptan for the treatment of hyponatremia in SCLC patients remain scarce. Furthermore, the most appropriate initial dose has not been identified. This study aimed to assess the effectiveness, safety, and survival rate associated with tolvaptan in regard to controlling hyponatremia in SCLC patients with the syndrome of inappropriate antidiuretic hormone secretion (SIADH) and to explore the appropriate initial dose.

Methods: A total of 23 SCLC patients with SIADH treated with tolvaptan were retrospectively reviewed between 2011 and 2019, and the efficacy and safety of tolvaptan were evaluated and compared between a $3.75 \mathrm{mg}$ dose and doses higher than $3.75 \mathrm{mg}$. Then, the Kaplan-Meier method was used to calculate overall survival (OS) and draw survival curves.

Results: In our center, patients had a mean age of $61.7 \pm 8.2$ years. The mean plasma sodium level after hypertonic saline treatment was $(120.7 \pm 7.5) \mathrm{mmol} / \mathrm{L}$. The doses of tolvaptan were distributed as follows: $3.75 \mathrm{mg}$ (10 patients), $5 \mathrm{mg}$ (5 patients), $7.5 \mathrm{mg}$ (4 patients), and $15 \mathrm{mg}$ (4 patients). After 3 days of tolvaptan treatment, the mean plasma sodium level was $(136.0 \pm 4.1) \mathrm{mmol} / \mathrm{L}$, which was significantly higher than the plasma sodium level on admission and before tolvaptan treatment $(\mathrm{P}<0.05)$. The mean time for tolvaptan to correct the plasma sodium level was $(3.7 \pm 5.8)$ days, and no significant difference was found between the $3.75 \mathrm{mg}$ group and the above $3.75 \mathrm{mg}$ group $(\mathrm{P}>0.05)$. Adverse events were observed in 1 patient who presented with severe lethargy and confusion. The median OS was 14.1 months.

Conclusions: In summary, no significant difference was found between the $3.75 \mathrm{mg}$ group and the above $3.75 \mathrm{mg}$ group. Less than $15 \mathrm{mg}$ of tolvaptan is enough to stabilize sodium levels for most patients. There is a risk of overcorrection of plasma sodium when using $15 \mathrm{mg}$ of tolvaptan. Tolvaptan can quickly improve performance status and may bring survival benefits to patients. The effect of tolvaptan in patients with SCLC and SIADH should be confirmed.

Keywords: Small cell lung cancer (SCLC); tolvaptan; hyponatremia; syndrome of inappropriate antidiuretic hormone hypersecretion (SIADH)

Submitted May 19, 2020. Accepted for publication Jan 29, 2021.

doi: $10.21037 /$ tcr-20-2123

View this article at: http://dx.doi.org/10.21037/tcr-20-2123

$\wedge$ ORCID: 0000-0002-1055-6753. 


\section{Introduction}

Small cell lung cancer (SCLC) is one of the most aggressive malignant tumors, with a five-year survival rate as low as $6.5 \%$ (1). The main symptoms of SCLC are respiratory disorders and paraneoplastic syndromes (2). Approximately $10 \%$ of lung cancer patients develop paraneoplastic syndromes (3), and the most common paraneoplastic syndrome in SCLC patients is the syndrome of inappropriate antidiuretic hormone secretion (SIADH).

SIADH is characterized by excessive unsuppressible release of antidiuretic hormone (4), unlike the concept of SIADH, the syndrome of inappropriate antidiuresis (SIAD) is a disorder of sodium and water balance characterized by urinary dilution impairment and hypotonic hyponatremia, in the absence of renal disease or any identifiable non-osmotic stimulus able to induce ADH release (5). The main clinical manifestation of SIADH is hyponatremia or a high urinary sodium concentration; hyponatremia occurs in approximately $15 \%$ of SCLC patients (6), which can be ascribed to SIADH in $60 \%$ of cases (7). Hyponatremia is defined as plasma sodium lower than $135 \mathrm{mmol} / \mathrm{L}$. According to the level of serum osmotic pressure, hyponatremia can be divided into hypertonic (>295 mOsm $/ \mathrm{kg})$, isotonic $(275-295 \mathrm{mOsm} / \mathrm{kg})$, and hypotonic $(<275 \mathrm{mOsm} / \mathrm{kg})$ conditions. Clinical symptoms include weakness, nausea and vomiting, headache and drowsiness, muscle pain and spasms, neuropsychiatric symptoms, and reversible ataxia, which depends on plasma sodium concentration (8). Hyponatremia is thought to be a negative prognostic factor in cancer patients based on a systematic analysis of published studies $(7,9,10)$. Hyponatremia and SIADH increase disease burden, prolong hospitalization, delay scheduled radiochemotherapy, and worsens patient Eastern Cooperative Oncology Group performance status (ECOG-PS) $(11,12)$.

SIADH-related hyponatremia can be improved with tumor regression through effective antitumor therapy, but poor performance status limits the possibility of chemoradiation. Recently, guidelines for managing hyponatremia in cancer patients have been established by several international panels $(5,13,14)$. These guidelines suggest that treatments for SIADH-related hyponatremia include fluid restriction, hypertonic saline, loop diuretics, isotonic saline, and tolvaptan. Only $5-14 \%$ of patients achieved plasma sodium levels above $130 \mathrm{mmol} / \mathrm{L}$ via fluid limitation and hypertonic saline; however, those therapies were often ineffective and sometimes even aggravated hyponatremia (15).

The SALT trials demonstrated the efficacy and safety of tolvaptan therapy in patients with hyponatremia resulting from multiple diseases, including SCLC with SIADH (16). However, the SALT trials did not show the SCLC subgroup results, and the clinical outcomes have not been reported. Previously, a prospective case series revealed that SCLC patients with SIADH responded well to $15 \mathrm{mg}$ of tolvaptan per day, and no serious adverse events or neuropsychiatric deficits were observed (17). However, three studies have reported that a $15 \mathrm{mg}$ dose of tolvaptan can overcorrect plasma sodium (18-20), and instead recommended an initial tolvaptan dose of $7.5 \mathrm{mg}$ for patients with SIADH. Additionally, pharmacodynamic studies performed by tolvaptan manufacturers have shown that $3.75 \mathrm{mg}$ can increase both urine output and plasma sodium levels (21).

To date, the largest study using tolvaptan to manage hyponatremia is SCLC patients with SIADH included only 10 patients (17). The remaining studies are case reports, and the most appropriate initial dose of tolvaptan has not been identified. To investigate the role of tolvaptan in managing hyponatremia in SCLC patients with SIADH in China and investigate the appropriate initial dose and clinical outcomes, we retrospectively reviewed 23 patients in our hospital. We present the following article in accordance with the STROBE reporting checklist (available at http:// dx.doi.org/10.21037/tcr-20-2123).

\section{Methods}

\section{Study design and participants}

From June 2011 to June 2019, 23 patients with SCLC and SIADH who received tolvaptan therapy were enrolled in this retrospective study at the Qilu Hospital, Cheeloo College of Medicine, Shandong University. Medical files were retrospectively collected on November 2019. The study was conducted in accordance with the Declaration of Helsinki (as revised in 2013). The study was approved by Qilu Hospital, Cheeloo College of Medicine, Shandong University (approval No. KYLL-2020-467) and individual consent for this retrospective analysis was waived.

\section{Patients}

Patients who received tolvaptan were analysed using data from medical records. The inclusion criteria were as follows: (I) patients were 18 years or older; (II) all patients 
had a histologically confirmed or cytologically confirmed diagnosis of SCLC; (III) patients had a clinical diagnosis of SIADH before tolvaptan therapy. The exclusion criteria were as follows: (I) patients with second primary tumors; (II) patients who received other treatments to correct hyponatremia while using tolvaptan; (III) patients complicated with other organ diseases; (IV) patients with hyponatremia due to other endocrine causes; (V) patients who had received antitumor therapy within three weeks. All patients were analysed in 23 patients who received tolvaptan.

\section{Protocol for hyponatremia correction}

The methods used to correct hyponatremia in this study were hypertonic saline and tolvaptan. The dose of $3 \%$ hypertonic saline solution (HTS) was calculated using the Adrogué-Madias formula (8). A rate of $20-30 \mathrm{~mL} / \mathrm{h}$ was used for intravenous infusion of HTS. Clinicians determined the dose and implementation of tolvaptan intervention based on the patient's condition and plasma sodium concentrations. The doses of tolvaptan were $3.75,5,7.5$, and $15 \mathrm{mg}$, and other treatments to correct plasma sodium were stopped 12 hours before initiating tolvaptan treatment. Plasma sodium concentrations were monitored at 12, 24, 48, and 72 hours ( 0 hours was the start of the drug intervention). The clinicians adjusted the treatment options based on plasma sodium concentrations.

\section{Study protocol}

The analysed data were as follows: age, gender, height, weight, body mass index (BMI), ECOG PS, cancer staging, time developed SIADH, plasma sodium at different periods, tolvaptan dose, effects of hyponatremia correction, length of follow up, and overall survival. For analysis, patients were grouped into a $3.75 \mathrm{mg}$ group $(\mathrm{n}=10)$ and a $>3.75 \mathrm{mg}$ group $(\mathrm{n}=13)$. Cancer staging was performed according to the Veterans Administration Lung Group (VALSG) 2-stage system and the American Joint Committee on Cancer's Cancer Staging Manual (eighth edition, 2018).

\section{Follow up}

After treatment, all patients were followed up through telephone interview every 3 months until death or last follow-up (2019/12/15).

\section{Statistical analysis}

Statistical analysis was performed with SPSS software (version 20.0; SPSS Inc., Chicago, IL, USA). Quantitative data are expressed as the mean \pm standard deviation; qualitative data are shown as n (\%). Exploratory analyses were performed to characterize the differences between the $3.75 \mathrm{mg}$ group and the above $3.75 \mathrm{mg}$ group. The correlation of categorical covariates was evaluated by Fisher's exact test, and the differences in continuous covariate models were evaluated by Student's $t$-test. Oneway analysis of variance (ANOVA) was used to assess plasma sodium levels according to different interventions. Overall survival was determined as the time from the date of SCLC diagnosis until death from any cause or the last follow-up. Then, the study used the Kaplan-Meier method to calculate overall survival and draw the survival curve. A P-value of less than 0.05 indicated statistical significance.

\section{Results}

\section{Demographic characteristics}

A total of 23 SCLC patients with SIADH were enrolled in this case series, and no patient was lost to follow-up. 10 of the 23 patients were first diagnosed with SCLC after hospitalization due to hyponatremia. The patient characteristics in Table 1 show that the patients (15 females, 8 males) had a mean age of $61.7 \pm 8.2$ years and an age range of 43-78 years. The mean height, weight, and BMI of the patients were $166.4 \pm 6.3,66.7 \pm 13.0$, and $24.0 \pm 4.2$, respectively. Eighteen (78.3\%) patients had limited disease, and $5(21.7 \%)$ patients had extensive disease. The ECOG PS scores were distributed as follows: 1 (1 patient), 2 (9 patients), and 3 (13 patients). The mean time of SIADH development was $(7.4 \pm 10.2)$ months. The mean baseline plasma sodium was $(122.4 \pm 6.5) \mathrm{mmol} / \mathrm{L}$, and the maximum value was $134 \mathrm{mmol} / \mathrm{L}$.

\section{The effect of hyponatremia correction and survival on SCLC patients}

During hyponatremia correction, 15 of 23 patients were treated with hypertonic saline after hospitalization; one patient achieved a plasma sodium level of $135 \mathrm{mmol} / \mathrm{L}$, and one patient had a plasma sodium level exceeding $135 \mathrm{mmol} /$ $\mathrm{L}$ after 30 days of tolvaptan treatment. The results in Table 2 show that the mean duration of hypertonic saline treatment 
Table 1 Characteristics of 23 patients with SCLC and SIADH

\begin{tabular}{|c|c|}
\hline Characteristic & Values (total $n=23$ ) \\
\hline Age (years) & $61.7 \pm 8.2$ \\
\hline Gender: (male/female) & $8(34.8) / 15(65.2)$ \\
\hline Height (cm) & $166.4 \pm 6.3$ \\
\hline Weight (kg) & $66.7 \pm 13.0$ \\
\hline BMI $\left(\mathrm{kg} / \mathrm{m}^{2}\right)$ & $24.0 \pm 4.2$ \\
\hline \multicolumn{2}{|l|}{ ECOG PS } \\
\hline $1 / 2 / 3$ & $1(4.3) / 9(39.1) / 13(56.5)$ \\
\hline \multicolumn{2}{|l|}{ T stage } \\
\hline $\mathrm{T} 1 / \mathrm{T} 2 / \mathrm{T} 3 / \mathrm{T} 4$ & $2(8.7) / 5(21.7) / 8(34.8) / 8(34.8)$ \\
\hline \multicolumn{2}{|l|}{$\mathrm{N}$ stage } \\
\hline N0/N1/N2/N3 & $7(30.4) / 7(30.4) / 7(30.4) / 2(8.7)$ \\
\hline \multicolumn{2}{|l|}{ M stage } \\
\hline Mo/M1 & $13(56.5) / 10(43.5)$ \\
\hline \multicolumn{2}{|l|}{ VALSG stage } \\
\hline LD/ED & $5(21.7) / 18(78.3)$ \\
\hline $\begin{array}{l}\text { Time to develop SIADH } \\
\text { (months) }\end{array}$ & $7.4 \pm 10.2$ \\
\hline $\begin{array}{l}\text { Plasma sodium on admission } \\
(\mathrm{mmol} / \mathrm{L})\end{array}$ & $122.4 \pm 6.5$ \\
\hline
\end{tabular}

Quantitative data were expressed as mean \pm SD; qualitative data were expressed by $n$ (\%). BMI, body mass index; ECOG PS, Eastern Cooperative Oncology Group performance status; TNM, tumor-node-metastasis; VALSG, Veterans Administration Lung Group; LD, limited disease; ED, extensive disease; SIADH, syndrome of inappropriate antidiuretic hormone secretion.

Table 2 The effect and safety of hyponatremia correction

\begin{tabular}{lc}
\hline Characteristic & Values (total $\mathrm{n}=23)$ \\
\hline HTS treatment time(days) & $5.0 \pm 7.4$ \\
Doses of tolvaptan & \\
$3.75 \mathrm{mg} / 5 \mathrm{mg} / 7.5 \mathrm{mg} / 15 \mathrm{mg}$ & $10(43.5) / 5(21.7) /$ \\
& $4(17.4) / 4(17.4)$ \\
Plasma sodium exceeds $135 \mathrm{mmol} / \mathrm{L}$ & $18(78.3) / 5(21.7)$ \\
after 3 days of tolvaptan (yes/no) & \\
Time to correct plasma sodium after & $3.7 \pm 5.8$ \\
treatment with tolvaptan (days) & \\
Adverse events & $1(4.3)$ \\
\hline
\end{tabular}

Quantitative data were expressed as mean $\pm \mathrm{SD}$; qualitative data were expressed by $n$ (\%). HTS, hypertonic saline solution. was $5.0 \pm 7.4$ days. The doses of tolvaptan were distributed as follows: $3.75 \mathrm{mg}$ (10 patients), $5 \mathrm{mg}$ (5 patients), $7.5 \mathrm{mg}$ (4 patients), and $15 \mathrm{mg}$ (4 patients). After treatment with tolvaptan, in 18 of 23 patients, the plasma sodium concentrations during the first three days exceeded $135 \mathrm{mmol} / \mathrm{L}$. In the remaining 5 patients, the plasma sodium concentration increased but did not reach $135 \mathrm{mmol} / \mathrm{L}$. The mean time to correct the plasma sodium level after tolvaptan treatment was $3.7 \pm 5.8$ days, and one patient had a plasma sodium level exceeding $135 \mathrm{mmol} / \mathrm{L}$ after 30 days of treatment. Adverse events were observed in 1 patient who presented with severe lethargy and confusion. The results in Figure 1 show that before tolvaptan treatment, the ECOG PS scores were distributed as follows: 1 (3 patients), 2 (10 patients), and 3 (10 patients), after 3 days of tolvaptan treatment, the ECOG PS scores were distributed as follows: 1 (3 patients), 2 (19 patients), and 3 (1 patient), which was a statistically significant difference between the two periods $(\mathrm{P}>0.05)$. On admission, the mean plasma sodium was $(122.4 \pm 6.5) \mathrm{mmol} / \mathrm{L}$, and the mean plasma sodium before tolvaptan treatment was $(120.7 \pm 7.5) \mathrm{mmol} / \mathrm{L}$. After 3 days of tolvaptan treatment, the mean plasma sodium was $136.0 \pm 4.1 \mathrm{mmol} / \mathrm{L}$, which was significantly higher than the plasma sodium on admission and before tolvaptan treatment $(\mathrm{P}<0.05)$.

Patients were stratified according to the dose of tolvaptan: the $3.75 \mathrm{mg}$ group [ $\mathrm{n}=10 ; 6$ males/ 4 females; mean age $(62.0 \pm 6.9)$ years] and the $>3.75 \mathrm{mg}$ group $[\mathrm{n}=13$; 9 males/4 females; mean age $(61.4 \pm 9.3)$ years]. Clinical data are shown in Table 3. On admission, there was no statistically significant difference in age, sex, height, weight, BMI, T stage, $\mathrm{N}$ stage, $\mathrm{M}$ stage, VALSG stage, plasma sodium, or ECOG PS $(\mathrm{P}>0.05)$ between the two groups. Similarly, there was no statistically significant difference in HTS intervention, duration of HTS treatment, or time to correct sodium by tolvaptan $(\mathrm{P}>0.05)$ between the two groups. Before tolvaptan treatment, there was no statistically significant difference in plasma sodium or ECOG PS $(P>0.05)$ between the two groups. After three days of tolvaptan treatment, there was no statistically significant difference in plasma sodium or ECOG PS $(\mathrm{P}>0.05)$ between the two groups.

The median OS was 14.1 months (Figure 2). The median follow-up time was 13 months (range, 3.8-30.0 months), and five patients were alive at the end of follow-up. No patient died from hyponatremia. 

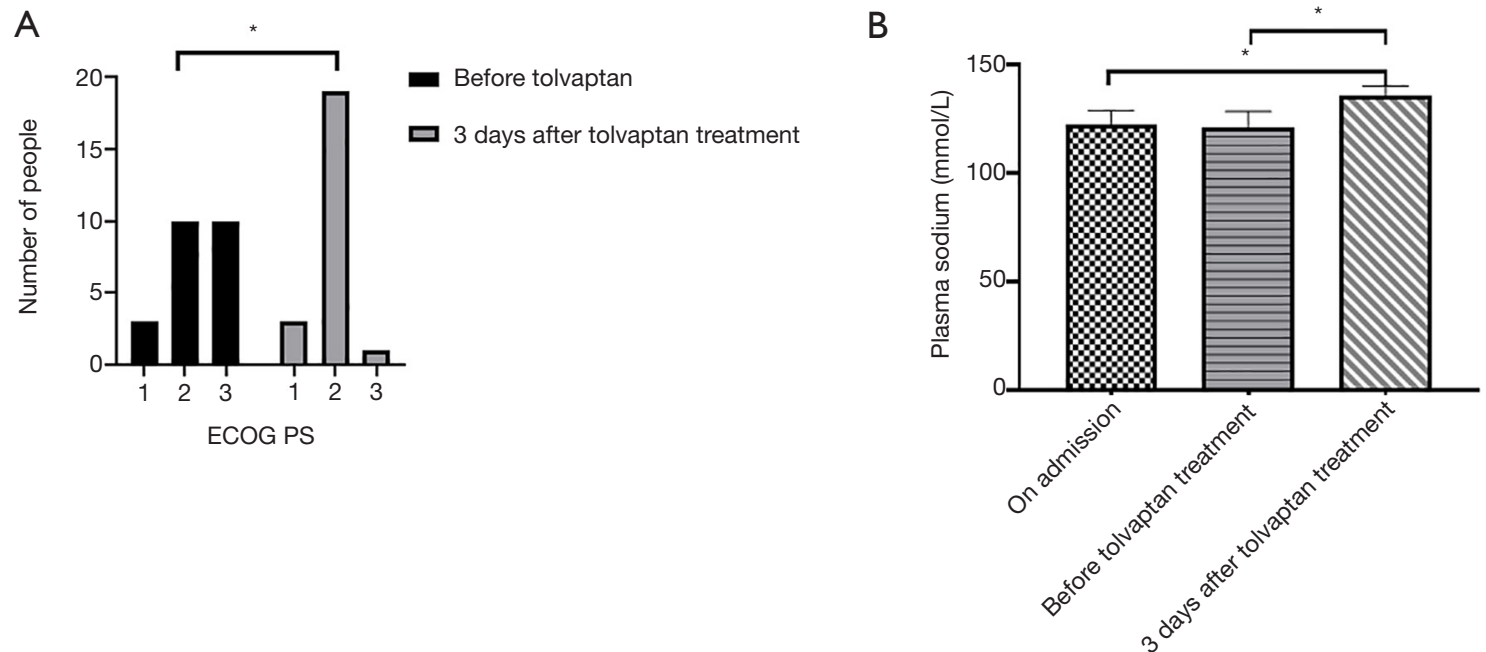

Figure 1 The effect of hyponatremia correction. (A) Distribution of ECOG PS scores in patients at different periods. (B) Comparison of serum sodium at the time of admission, before tolvaptan treatment, and three days after tolvaptan treatment showed that patients had significantly higher plasma sodium after 3 days of tolvaptan treatment. *, $\mathrm{P}<0.05$. ECOG PS, Eastern Cooperative Oncology Group performance status.

\section{Discussion}

Hyponatremia related to SIADH is an essential complication in SCLC patients. In China, tolvaptan was indicated for clinically significant euvolemic and hypervolemic hyponatremia, including patients with heart failure, liver cirrhosis, and SIADH (22). In recent decades, many studies have shown Tolvaptan to be effective in correcting plasma sodium (11-16). Petereit et al. (17) summarized the characteristics of 10 patients with SCLC and SIADH in the largest observational study that we found. To the best of our knowledge, our study is the largest monocentric series of SCLC patients with SIADH. Our study found that tolvaptan at a dose of less than $15 \mathrm{mg}$ is safe and effective for most patients. We also observed doserelated effectiveness and safety issues in individual patients. In addition, patient survival may benefit from tolvaptan treatment.

In most cases, SIADH associated with SCLC arises from the ectopic release of ADH/ADH - like substances from tumor cells (23). Hyponatremia secondary to cancer may persist for a long time in the absence of effective antitumor therapies to reduce the tumor burden. In addition, some studies have confirmed that hypertonic saline may be ineffective for SIADH treatment and may even exacerbate hyponatremia $(15,24)$. Petereit et al. demonstrated the effectiveness of tolvaptan in correcting hyponatremia in
SCLC patients with SIADH, where the plasma sodium levels increased significantly in the majority of patients within four days (17); although two patients still needed more than 10 days of treatment. In our retrospective study, only one patient was successfully treated with hypertonic saline. The mean time to correct plasma sodium after tolvaptan treatment was $3.7 \pm 5.8$, and after 3 days of tolvaptan treatment, the mean plasma sodium level was $136.0 \pm 4.1 \mathrm{mmol} / \mathrm{L}$; however, one patient took 30 days of treatment to correct hyponatremia. Whether this was due to the lower dose of oral tolvaptan $(5 \mathrm{mg})$ requires further investigation.

So far, no consensus has been reached about the initial dose of tolvaptan for SIADH treatment $(18,19,21,25)$. A previous retrospective study concluded that the recommended initial dose of tolvaptan for the treatment of hyponatremia ranges from 15 to $60 \mathrm{mg} /$ day (26). A study involving 13 patients with SIADH, including 7 SCLC patients, showed that in 11 patients, taking $7.5 \mathrm{mg}$ of tolvaptan alone on alternate days corrected plasma sodium levels (27). Similarly, in one RCT, Castello et al. treated patients with hypervolemic or euvolemic hyponatremia using 7.5 or $15 \mathrm{mg}$ tolvaptan, and the authors found that $7.5 \mathrm{mg}$ tolvaptan was both effective and safe; compared to $7.5 \mathrm{mg}, 15 \mathrm{mg}$ tolvaptan had a risk for sodium overcorrection (18). A South Korean multicenter study involving 51 patients with SIADH concluded that 
Table 3 The effects of tolvaptan in the $3.75 \mathrm{mg}$ group and $>3.75 \mathrm{mg}$ group

\begin{tabular}{|c|c|c|c|}
\hline Characteristic & 3.75 mg group $(n=10)$ & $>3.75$ mg group $(n=13)$ & $\mathrm{P}$ \\
\hline Gender (male/female) & $6 / 4$ & $9 / 4$ & 0.685 \\
\hline Weight (kg) & $69.3 \pm 13.7$ & $64.62 \pm 12.6$ & 0.849 \\
\hline Height (cm) & $165.5 \pm 7.5$ & $167.1 \pm 5.4$ & 0.122 \\
\hline T stage $(1 / 2 / 3 / 4)$ & $1 / 2 / 5 / 2$ & $1 / 3 / 3 / 6$ & 0.503 \\
\hline $\mathrm{N}$ stage $(0 / 1 / 2 / 3)$ & $3 / 5 / 2 / 0$ & $4 / 2 / 5 / 2$ & 0.222 \\
\hline M stage $(0 / 1)$ & $8 / 2$ & $5 / 8$ & 0.09 \\
\hline VALSG stage (LD/ED) & $4 / 6$ & $1 / 12$ & 0.127 \\
\hline Plasma sodium (mmol/L) & $121.6 \pm 6.9$ & $123.0 \pm 6.4$ & 0.964 \\
\hline HTS Intervention (yes/no) & $4 / 6$ & $4 / 9$ & 0.685 \\
\hline Duration of HTS treatment (days) & $2.9 \pm 3.4$ & $6.6 \pm 9.2$ & 0.113 \\
\hline \multicolumn{4}{|l|}{ Before tolvaptan treatment } \\
\hline ECOG PS (1/2/3) & $1 / 6 / 3$ & $2 / 4 / 7$ & 0.372 \\
\hline Plasma sodium (mmol/L) & $122.0 \pm 7.0$ & $120.9 \pm 7.5$ & 0.91 \\
\hline Time to correct sodium by tolvaptan (days) & $2.7 \pm 0.8$ & $4.46 \pm 7.8$ & 0.138 \\
\hline \multicolumn{4}{|l|}{ After 3 days of tolvaptan treatment } \\
\hline
\end{tabular}

Quantitative data were expressed as mean \pm SD; qualitative data were expressed by $n$ (\%). HTS, hypertonic saline solution; ECOG PS, Eastern Cooperative Oncology Group performance status; TNM, tumor-node-metastasis; VALSG, Veterans Administration Lung Group; LD, limited disease; ED, extensive disease.

$15 \mathrm{mg}$ of tolvaptan was associated with a high incidence of overcorrection, and the authors suggested that Koreans use $7.5 \mathrm{mg}$ tolvaptan as the initial dose (19). In our study, the majority of patients that were administered low doses of tolvaptan showed adequate safety and efficacy. Among the 4 patients who received $15 \mathrm{mg}$ of tolvaptan, the plasma sodium level of one patient increased from 119 to $142 \mathrm{mmol} / \mathrm{L}$ within the first 24 hours, and lethargy and unconsciousness were observed. Thus, the recommended initial dose of tolvaptan will require that this safety issue be solved.

A post-marketing evaluation of tolvaptan in Europe found that doctors often split or crush the tablets to make a lower initial dose. Manufacturers' pharmacodynamic studies have shown that both 3.75 and $7.5 \mathrm{mg}$ doses can increase urine output and increase plasma sodium levels, while the $7.5 \mathrm{mg}$ dose can lead to overcorrection (21). No difference was found in our study between the $3.75 \mathrm{mg}$ group and the above $3.75 \mathrm{mg}$ group, our research indicates that $3.75 \mathrm{mg}$ is the proper initial dose of tolvaptan in treating SCLC with SIADH.

Interest in the initial dose has focused on sodium overcorrection in hyponatremic patients treated with $15 \mathrm{mg}$ tolvaptan. Two guidelines recommended that a limit to hyponatremia correction is an increase in sodium concentration of $<10-12 \mathrm{mmol} / \mathrm{L}$ in $24 \mathrm{~h}$ and of $<18 \mathrm{mmol} / \mathrm{L}$ in $48 \mathrm{~h}(13,28)$. Overcorrection of hyponatremia is defined as a rapid increase in plasma sodium 


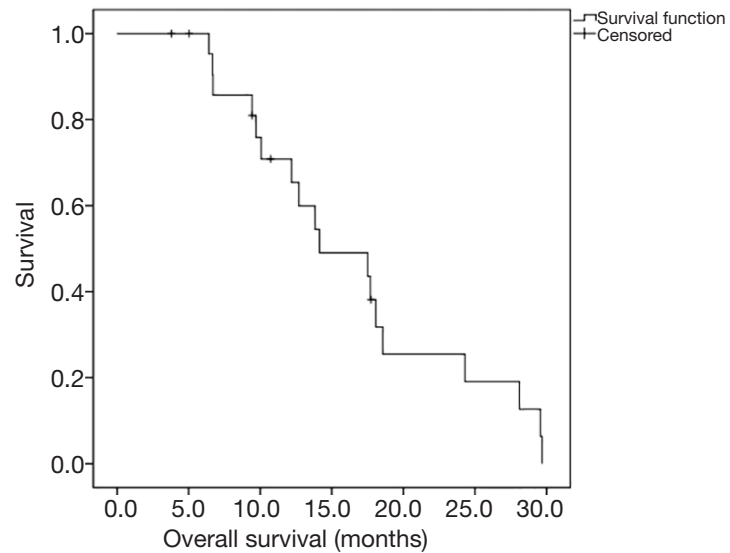

Figure 2 Survival curve of 23 SCLC patients with inappropriate antidiuretic hormone. The median overall survival was 14.1 months.

concentration, which can lead to severe complications such as osmotic demyelination syndrome (ODS) associated with high patient mortality (28).

In three studies, the incidence of overcorrection caused by tolvaptan ranged from $4.5 \%$ to $31.2 \%$ (29-31). Previous studies have demonstrated that overcorrection caused by tolvaptan was associated with lower plasma sodium concentrations at baseline (30,32-35). A retrospective study of tolvaptan in South Korea found that overcorrection of hyponatremia was associated with lower BMI, the presence of cancer, and lower plasma sodium at baseline; in the SIADH subpopulation, baseline sodium and cancer were significant key factors associated with overcorrection $(34,35)$. In our study, one patient experienced lethargy and unconsciousness and a decline in performance status due to sodium overcorrection. It is well known that hyponatremia has a negative influence on the performance status of patients $(17,36,37)$, and few studies have confirmed that tolvaptan improved ECOG performance status in patients with SCLC and SIADH (17).

In our study, the PS of the majority of patients improved during tolvaptan treatment. Improving the performance status of cancer patients allows patients to receive radiotherapy and chemotherapy on time. The negative effects of delayed treatment could thus be avoided. However, it is unclear whether this effect can bring survival benefits. The presence of hyponatremia is a factor associated with poor prognosis of SCLC. From a retrospective study involving 395 SCLC patients, Hermes et al. concluded that the hyponatremia subgroup showed poor median survival
(9.0 vs. 13.0 months, $\mathrm{P}<0.001$ ) when compared to that of the normal sodium subgroup (38). Similar conclusions were drawn in another retrospective study involving 453 SCLC patients conducted by Hansen et al., in which the hyponatremia subgroup showed worse median survival (7.1 vs. 11.2 months, $\mathrm{P}=0.0001$ ) (7). In our study, the median survival of patients after the administration of tolvaptan was 14.1 months. This median survival seems longer than the previously reported values ranging from 7.1 to 13.0 months $(7,38)$. No survival data on patients with SCLC and SIADH treated with oral tolvaptan can be compared, and we cannot rule out the possibility that this discrepancy is due to insufficient sample size.

This study has the following limitations: first, the use of tolvaptan in China has not yet become widespread, resulting in a small sample size; second, because this was a retrospective study, time data were not accurate to the hour; third, the data were summarized from a single institution, making it difficult to form general conclusions. We suggest a series of remedy, multicenter, prospective, randomized controlled experiments in future studies.

\section{Conclusions}

In summary, our study shows that using a dose of tolvaptan lower than $15 \mathrm{mg}$ is effective in stabilizing sodium levels for most patients. There is a risk of overcorrection of plasma sodium when using $15 \mathrm{mg}$ of tolvaptan. Tolvaptan can quickly improve the performance status of patients, which may bring survival benefits to patients. The effect of tolvaptan on SCLC patients with SIADH should be confirmed.

\section{Acknowledgments}

We would like to thank Alvin Han, undergraduate student, Department of Biochemistry and Biomedical Sciences, McMaster University, for his editing on written expression and grammar of this manuscript.

Funding: This study was funded by Shandong University Joint Foundation "Resistance mechanism and optimal treatment for advanced Lung Cancer" (Grant No. 26010112671829)

\section{Footnote}

Reporting Checklist: The authors have completed the STROBE reporting checklist. Available at http://dx.doi. 
org/10.21037/tcr-20-2123

Data Sharing Statement: Available at http://dx.doi. org/10.21037/tcr-20-2123

Peer Review File: Available at http://dx.doi.org/10.21037/tcr20-2123

Conflicts of Interest: All authors have completed the ICMJE uniform disclosure form (available at http://dx.doi. org/10.21037/tcr-20-2123). The authors have no conflicts of interest to declare.

Ethical Statement: The authors are accountable for all aspects of the work in ensuring that questions related to the accuracy or integrity of any part of the work are appropriately investigated and resolved. The study was conducted in accordance with the Declaration of Helsinki (as revised in 2013). The study was approved by Qilu Hospital, Cheeloo College of Medicine, Shandong University (approval No. KYLL-2020-467). and individual consent for this retrospective analysis was waived.

Open Access Statement: This is an Open Access article distributed in accordance with the Creative Commons Attribution-NonCommercial-NoDerivs 4.0 International License (CC BY-NC-ND 4.0), which permits the noncommercial replication and distribution of the article with the strict proviso that no changes or edits are made and the original work is properly cited (including links to both the formal publication through the relevant DOI and the license). See: https://creativecommons.org/licenses/by-nc-nd/4.0/.

\section{References}

1. Howlader N, Noone AM, Krapcho M, et al. SEER Cancer Statistics Review, 1975-2016, National Cancer Institute.

Bethesda, MD. Available online: https://seer.cancer.gov/ csr/1975_2016/

2. Raspotnig M, Vedeler C, Storstein A. Paraneoplastic neurological syndromes in lung cancer patients with or without onconeural antibodies. J Neurol Sci 2015;348:41-5.

3. Spiro SG, Gould MK, Colice GL. Initial evaluation of the patient with lung cancer: symptoms, signs, laboratory tests, and paraneoplastic syndromes: ACCP evidencedbased clinical practice guidelines (2nd edition). Chest 2007;132:S149-60.
4. Babar SM. SIADH associated with ciprofloxacin. Ann Pharmacother 2013;47:1359-63.

5. Esposito P, Piotti G, Bianzina S, et al. The syndrome of inappropriate antidiuresis: pathophysiology, clinical management and new therapeutic options. Nephron Clin Pract 2011;119:c62-73.

6. Castillo JJ, Vincent M, Justice E. Diagnosis and management of hyponatremia in cancer patients. Oncologist 2012;17:756-65.

7. Hansen O, Sørensen P, Hansen KH. The occurrence of hyponatremia in SCLC and the influence on prognosis: a retrospective study of 453 patients treated in a single institution in a 10-year period. Lung Cancer 2010;68:111-4.

8. Adrogué HJ, Madias NE. Hyponatremia. N Engl J Med 2000;342:1581-9.

9. Petereit C, Zaba O, Teber I, et al. Is hyponatremia a prognostic marker of survival for lung cancer? Pneumologie 2011;65:565-71.

10. Iyer AV, Krasnow SH, Dufour DR, et al. Sodium-wasting nephropathy caused by cisplatin in a patient with small-cell lung cancer. Clin Lung Cancer 2003;5:187-9.

11. Berardi R, Caramanti M, Castagnani M, et al. Hyponatremia is a predictor of hospital length and cost of stay and outcome in cancer patients. Support Care Cancer 2015;23:3095-101.

12. Doshi SM, Shah P, Lei X, et al. Hyponatremia in hospitalized cancer patients and its impact on clinical outcomes. Am J Kidney Dis 2012;59:222-8.

13. Verbalis JG, Goldsmith SR, Greenberg A, et al. Diagnosis, evaluation, and treatment of hyponatremia: expert panel recommendations. Am J Med 2013;126:S1-42.

14. Runkle I, Villabona C, Navarro A, et al. Treatment of hyponatremia induced by the syndrome of Inappropriate antidiuretic hormone secretion: a multidisciplinary Spanish algorithm. Nefrologia 2014;34:439-50.

15. Burst V, Grundmann F, Kubacki T, et al. Euvolemic hyponatremia in cancer patients. Report of the Hyponatremia Registry: an observational multicenter international study. Support Care Cancer 2017;25:2275-83.

16. Verbalis JG, Adler S, Schrier RW, et al. Efficacy and safety of oral tolvaptan therapy in patients with the syndrome of inappropriate antidiuretic hormone secretion. Eur J Endocrinol 2011;164:725-32.

17. Petereit C, Zaba O, Teber I, et al. A rapid and efficient way to manage hyponatremia in patients with SIADH and small cell lung cancer: treatment with tolvaptan. BMC 
Pulm Med 2013;13:55.

18. Castello LM, Baldrighi M, Panizza A, et al. Efficacy and safety of two different tolvaptan doses in the treatment of hyponatremia in the Emergency Department. Intern Emerg Med 2017;12:993-1001.

19. Han SW, Yi JH, Kang KP, et al. Safety and Efficacy of Tolvaptan in Korean Patients with Hyponatremia Caused by the Syndrome of Inappropriate Antidiuretic Hormone. J Korean Med Sci 2018;33:e112.

20. Tzoulis P, Waung JA, Bagkeris E, et al. Real-life experience of tolvaptan use in the treatment of severe hyponatraemia due to syndrome of inappropriate antidiuretic hormone secretion. Clin Endocrinol (Oxf) 2016;84:620-6.

21. Shoaf SE, Bricmont P, Dandurand A. Low-dose tolvaptan PK/PD: comparison of patients with hyponatremia due to syndrome of inappropriate antidiuretic hormone secretion to healthy adults. Eur J Clin Pharmacol 2017;73:1399-408.

22. Chen S, Zhao JJ, Tong NW, et al. Randomized, double blinded, placebo-controlled trial to evaluate the efficacy and safety of tolvaptan in Chinese patients with hyponatremia caused by SIADH. J Clin Pharmacol 2014;54:1362-7.

23. Grohé C, Berardi R, Burst V. Hyponatraemia-SIADH in lung cancer diagnostic and treatment algorithms. Crit Rev Oncol Hematol 2015;96:1-8.

24. Schwartz WB, Bennett W, Curelop S, et al. A syndrome of renal sodium loss and hyponatremia probably resulting from inappropriate secretion of antidiuretic hormone. Am J Med 1957;23:529-42.

25. Harbeck B, Lindner U, Haas CS. Low-dose tolvaptan for the treatment of hyponatremia in the syndrome of inappropriate $\mathrm{ADH}$ secretion (SIADH). Endocrine 2016;53:872-3.

26. Sahay M, Sahay R. Hyponatremia: a practical approach. Indian J Endocrinol Metab 2014;18:760-71.

27. Kenz S, Haas CS, Werth SC, et al. High sensitivity to tolvaptan in paraneoplastic syndrome of inappropriate ADH secretion (SIADH). Ann Oncol 2011;22:2696.

28. Spasovski G, Vanholder R, Allolio B, et al. Clinical practice guideline on diagnosis and treatment of hyponatraemia.

Cite this article as: Ren P, Yang Q. The role of tolvaptan in managing hyponatremia in small cell lung cancer patients with SIADH: a retrospective study of 23 cases. Transl Cancer Res 2021;10(3):1229-1237. doi: 10.21037/tcr-20-2123
Eur J Endocrinol 2014;170:G1-47.

29. Aratani S, Hara M, Nagahama M, et al. A low initial serum sodium level is associated with an increased risk of overcorrection in patients with chronic profound hyponatremia: a retrospective cohort analysis. BMC Nephrol 2017;18:316.

30. Umbrello M, Mantovani ES, Formenti P, et al. Tolvaptan for hyponatremia with preserved sodium pool in critically ill patients. Ann Intensive Care 2016;6:1.

31. Kim Y, Lee N, Lee KE, et al. Risk factors for sodium overcorrection in non-hypovolemic hyponatremia patients treated with tolvaptan. Eur J Clin Pharmacol 2020;76:723-9.

32. Vaghasiya RP, DeVita MV, Michelis MF. Serum and urine responses to the aquaretic agent tolvaptan in hospitalized hyponatremic patients. Int Urol Nephrol 2012;44:865-71.

33. Gheorghiade M, Konstam MA, Burnett JC Jr, et al. Short-term clinical effects of tolvaptan, an oral vasopressin antagonist, in patients hospitalized for heart failure: the EVEREST Clinical Status Trials. JAMA 2007;297:1332-43.

34. Hirai K, Shimomura T, Moriwaki H, et al. Risk factors for hypernatremia in patients with short- and longterm tolvaptan treatment. Eur J Clin Pharmacol 2016;72:1177-83.

35. Morris JH, Bohm NM, Nemecek BD, et al. Rapidity of Correction of Hyponatremia Due to Syndrome of Inappropriate Secretion of Antidiuretic Hormone Following Tolvaptan. Am J Kidney Dis 2018;71:772-82.

36. Jacot W, Colinet B, Bertrand D, et al. Quality of life and comorbidity score as prognostic determinants in non-small-cell lung cancer patients. Ann Oncol 2008;19:1458-64.

37. Sengupta A, Banerjee SN, Biswas NM, et al. The Incidence of Hyponatraemia and Its Effect on the ECOG Performance Status among Lung Cancer Patients. J Clin Diagn Res 2013;7:1678-82.

38. Hermes A, Waschki B, Reck M. Hyponatremia as prognostic factor in small cell lung cancer--a retrospective single institution analysis. Respir Med 2012;106:900-4. 